\title{
Time-resolved spectroscopy on GaN nanocolumns grown by plasma assisted molecular beam epitaxy on Si substrates
}

\author{
P. Corfdir, ${ }^{1, a)}$ P. Lefebvre, ${ }_{1}^{2,1}$ J. Ristić, ${ }^{1,3}$ P. Valvin, ${ }^{2}$ E. Calleja, ${ }^{3}$ A. Trampert, ${ }^{4}$ J.-D. Ganière, ${ }^{1}$ \\ and B. Deveaud-Plédran ${ }^{1}$ \\ ${ }^{1}$ Institut de Photonique et d'Electronique Quantiques, Ecole Polytechnique Fédérale de Lausanne (EPFL), \\ 1015 Lausanne, Switzerland \\ ${ }^{2}$ Groupe d'Etude des Semiconducteurs (GES)-CNRS_UMR5650-Université Montpellier II, Case Courrier \\ 074. F-34095 Montpellier Cedex 5, France \\ ${ }^{3}$ Departamento de Ingeniería Electrónica and ISOM, ETSI Telecomunicación, Universidad Politécnica, \\ 28040 Madrid, Spain \\ ${ }^{4}$ Paul-Drude-Institut für Festkörperelektronik, Hausvogteiplatz 5-7, D-10117 Berlin, Germany
}

(Received 9 October 2008; accepted 18 November 2008; published online 13 January 2009)

\begin{abstract}
A detailed study of excitons in unstrained $\mathrm{GaN}$ nanocolumns grown by plasma assisted molecular beam epitaxy on silicon substrates is presented. The time-integrated and time-resolved photoluminescence spectra do not depend significantly on the (111) or (001) Si surface used. However, an unusually high relative intensity of the two-electron satellite peak of the dominant donor-bound exciton line is systematically observed. We correlate this observation with the nanocolumn morphology determined by scanning electron microscopy, and therefore propose an interpretation based on the alteration of wave functions of excitonic complexes and of donor states by the proximity of the semiconductor surface. This explanation is supported by a model that qualitatively accounts for both relative intensities and time decays of the photoluminescence lines. (C) 2009 American Institute of Physics. [DOI: 10.1063/1.3062742]
\end{abstract}

\section{INTRODUCTION}

Since Nakamura et al. ${ }^{1}$ reported in 1994 the fabrication of blue-light-emitting diodes, $\mathrm{GaN}$ and related alloys have attracted a great interest of the scientific community, giving way to a huge improvement of material quality and to the fabrication of a wide variety of electronic and optoelectronic devices. However, the lack of lattice matched substrates remains one of the bottlenecks for a wider range of applications of nitride based devices. Indeed, heteroepitaxial growth results in large densities of extended defects ${ }^{2}$ that deteriorate the performances and affect the lifetime of the devices. One alternative recently proposed to overcome this problem is based on the fabrication of monocrystalline nitride nanocolumns (NCs). Semiconductor NC heterostructures are expected to promote a variety of possible device applications, some having already been demonstrated: nanowire lasers, light-emitting diodes, ${ }^{4}$ or transistors. ${ }^{5}$

Plasma assisted molecular beam epitaxy (PAMBE) grown GaN NCs with diameters from 30 to $150 \mathrm{~nm}$ and about $1 \mu \mathrm{m}$ high were proven to be dislocation- and strainfree structures. ${ }^{6-8}$ Alternative synthesis techniques are hydride vapor phase epitaxy ${ }^{9}$ or metal-organic chemical vapor deposition. ${ }^{10} \mathrm{NCs}$ made of $\mathrm{GaN},(\mathrm{Al}, \mathrm{Ga}) \mathrm{N}$, and (In, Ga)N as well as their heterostructures ${ }^{11,12}$ can be grown on a wide variety of substrates, such as $\mathrm{Si}(001),{ }^{13} \mathrm{Si}(111),{ }^{8}$ and sapphire, ${ }^{6,7,14}$ with or without GaN buffer layer, the $c$-axis of the columns being perpendicular to the surface of the substrate. Even though the activity in this field has been quite significant in the past decade, rather little attention has been devoted to the detailed study of optical properties of pure

${ }^{a)}$ Electronic mail: pierre.corfdir@epfl.ch.
GaN NCs, in relation to their particular geometry. Indeed, most optical studies were aimed at characterizing the crystalline and chemical quality of the NCs since quantum confinement effects were neither expected nor convincingly observed, due to the large diameter of the grown NCs, compared to the excitonic Bohr radius.

This work presents the results of time-integrated and time-resolved photoluminescence (TI- and TR-PL) studies performed on GaN NCs grown on Si substrates. We investigate and discuss some original features of excitonic emission spectra that differ from those of GaN compact epilayers.

\section{SAMPLES AND EXPERIMENTAL DETAILS}

The studied GaN NCs have been grown by radiofrequency PAMBE on bare $\mathrm{Si}(111)$ and $\mathrm{Si}$ (001) substrates far below stoichiometric conditions (III/V ratio $\ll 1)$. By varying the III/V ratio during the growth, one controls the density and the diameter of these self-ordered nanostructures: ${ }^{6,15}$ the smaller the III/V ratio, the smaller the diameter, and the higher the density of NCs. The exceptional crystalline quality of the NCs has been verified previously by transmission electron microscopy ${ }^{16}$ and Raman scattering studies, ${ }^{8}$ also proving that they are strain-free. Concerning their morphology, Fig. 1 shows scanning electron microscopy (SEM) micrographs for two samples, together with the corresponding continuous-wave PL spectra. In general, SEM reveals arrays of well separated NCs, aligned along the $c$ direction, whatever crystallographic plane of the substrate used. Such measurements provided proper estimation of the average NC radii (see Table I). Figure 1 also suggests a possible correlation between the PL spectra and the sample morphology. We will discuss this issue further on in this work. 

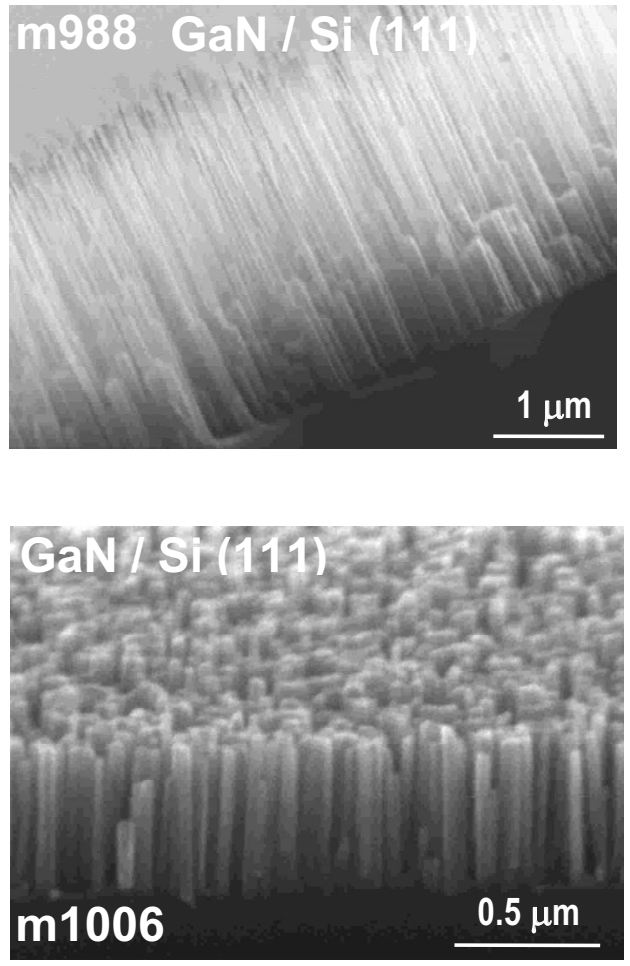
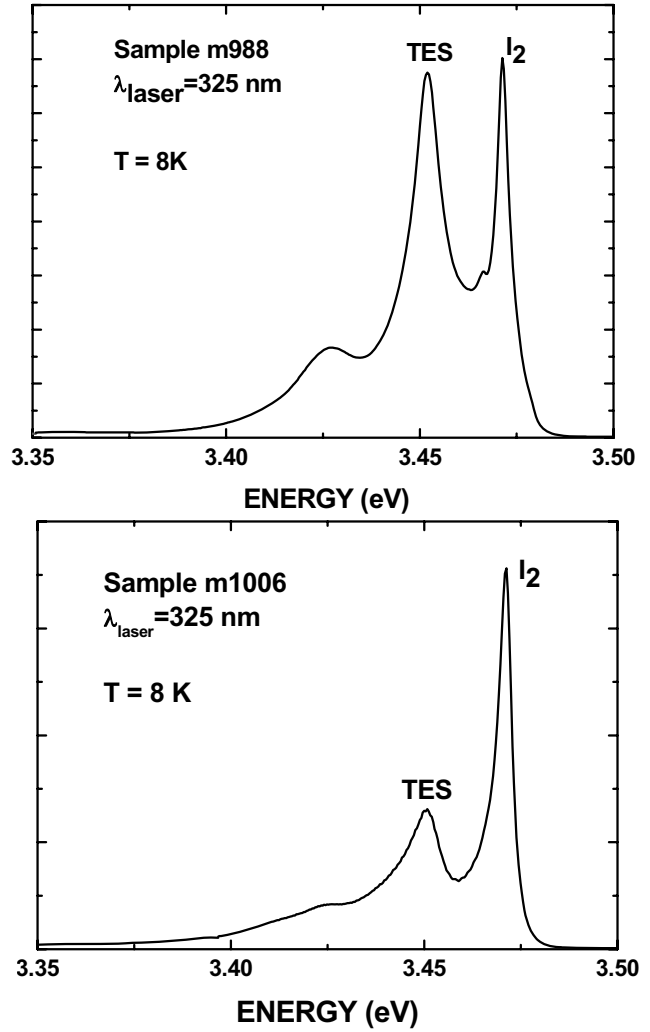

FIG. 1. SEM images of GaN NCs grown on Si(111) substrates (samples m988 and m1006), with respective continuous-wave PL spectra excited by laser radiation at $325 \mathrm{~nm}$. The NC diameters are much smaller in sample m988, and the PL line at $3.45 \mathrm{eV}$ is also much more intense than for sample m1006.

It must be emphasized that no differences in structural and optical properties between the NCs grown on Si (100) and $\mathrm{Si}(111)^{13}$ were observed. Therefore, we will discuss from now on the optical properties of the NCs without distinction of the substrate orientation.

Our TR-PL setup uses the third harmonic of an $\mathrm{Al}_{2} \mathrm{O}_{3}: \mathrm{Ti}$ mode-locked laser $(\lambda=280 \mathrm{~nm})$, with pulse width and repetition rate of $2 \mathrm{ps}$ and $80.7 \mathrm{MHz}$, respectively. The samples are cooled in a closed-cycle He cryostat down to 8K. TR-PL spectra were analyzed by a monochromator (grating of either 1200 or 600 grooves $/ \mathrm{mm}$ ), followed by a streak camera and using a photon counting mode.

\section{TIME-INTEGRATED PHOTOLUMINESCENCE}

Figure 2 displays the TI-PL spectra taken between 8 and $200 \mathrm{~K}$ for sample m1253. The spectrum at $8 \mathrm{~K}$ is typical of all studied samples in terms of PL peak energies. What really changes among these samples is the intensity ratio between the dominant lines, at $3470.0 \pm 0.1$ and $3448.5 \pm 0.2 \mathrm{meV}$. In addition, weaker lines are observed at $3476.9 \pm 0.4$ and $3485 \pm 1 \mathrm{meV}$. The relative increase in intensity of these higher energy lines with temperature with respect to the line at $3470.0 \mathrm{meV}$ allows us to attribute them to free excitons $A$ $\left(\mathrm{FX}_{A}\right)$ and $B\left(\mathrm{FX}_{B}\right)$, respectively. The peak at $3470.0 \mathrm{meV}$ is then readily assigned to the so-called $\mathrm{I}_{2}$ recombination of $A$-exciton bound to a neutral donor $\left(\mathrm{D}^{\circ} \mathrm{X}\right)$. As already noticed, ${ }^{6}$ these energies for bound and free exciton transitions correspond to fully unstrained $\mathrm{GaN},{ }^{17}$ which seems contradictory with the somewhat large linewidths [full width at half maximum (FWHM) of $\sim 5 \mathrm{meV}$, typically] observed. We will comment later in this work that these linewidths may have an intrinsic origin, not related to strain. Due to these linewidth values, it is difficult to assign the $\mathrm{I}_{2}$ line to a specific donor ( $\mathrm{Si}$ or $\mathrm{O}$ ). Nevertheless, we assume that the dominant donor be silicon, ${ }^{18,19}$ given the nature of the substrate. In fact, between lattice temperatures of 15 and $45 \mathrm{~K}$, due to

TABLE I. Characteristics of the GaN NC samples, including the average radii measured by SEM and the intensities and decay times measured by TR-PL. The results of the fitting procedure by our two-zone (core-shell) modeling are given in the last five columns.

\begin{tabular}{|c|c|c|c|c|c|c|c|c|c|c|}
\hline \multicolumn{2}{|l|}{ Samples } & \multicolumn{4}{|c|}{ Expt. results } & \multicolumn{5}{|c|}{ Calc./Fitting } \\
\hline Name & Si substrate & $\begin{array}{l}\text { Radius } \\
(\mathrm{nm})\end{array}$ & $\rho=\frac{I_{\mathrm{I}_{2}}(0)}{I_{\mathrm{TES}}(0)}$ & $\begin{array}{c}\tau_{\mathrm{PL}}\left(\mathrm{I}_{2}\right) \\
(\mathrm{ns})\end{array}$ & $\begin{array}{c}\tau_{\mathrm{PL}}(\mathrm{TES}) \\
(\mathrm{ns})\end{array}$ & $\alpha$ & $\begin{array}{l}\tau_{2(s)} \\
(\mathrm{ns})\end{array}$ & $\begin{array}{c}\tau_{2(c)}=\underset{(\mathrm{ns})}{50 \times \tau_{1(c)}} \\
\end{array}$ & $\begin{array}{l}\tau_{1(c)} \\
(\mathrm{ns})\end{array}$ & $\begin{array}{l}\tau_{1(s)} \\
(\mathrm{ns})\end{array}$ \\
\hline m988 & (111) & $15 \pm 3$ & 1.1 & $0.12 \pm 0.02$ & $0.41 \pm 0.02$ & 0.17 & 0.5 & 5.1 & 0.10 & 2.8 \\
\hline m1006 & (111) & $24 \pm 4$ & 2.2 & $0.16 \pm 0.02$ & $0.32 \pm 0.02$ & 0.40 & 0.4 & 6.9 & 0.14 & 1.5 \\
\hline $\mathrm{m} 1253$ & (111) & $25 \pm 4$ & 1.9 & $0.15 \pm 0.02$ & $0.34 \pm 0.03$ & 0.35 & 0.4 & 7.0 & 0.14 & 1.6 \\
\hline $\mathrm{m} 1254$ & (100) & $34 \pm 5$ & 8.6 & $0.20 \pm 0.02$ & $0.37 \pm 0.02$ & 0.57 & 1.0 & 9.5 & 0.19 & 0.6 \\
\hline
\end{tabular}




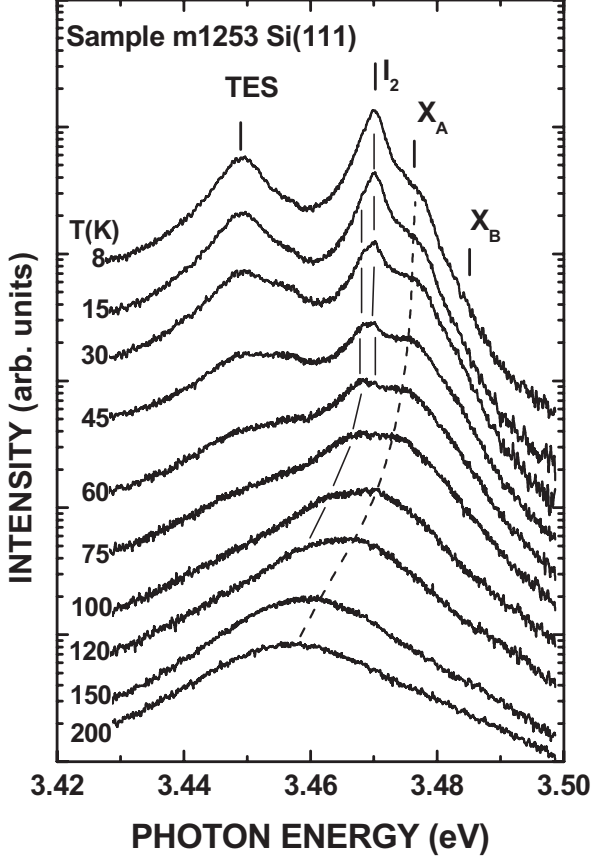

FIG. 2. Evolution with temperature of the TI PL spectrum of sample m1253, in the excitonic region. The logarithmic scale permits the observation of free exciton transitions, at 3.477 and $3.485 \mathrm{eV}$. Segments and dashed lines are guides to the eyes, emphasizing, for instance, the double structure of the $\mathrm{I}_{2}$ line

less efficient thermal detrapping of the exciton, a second line appears on the low-energy side of the main $\mathrm{I}_{2}$ line that certainly relates to a deeper donor.

Concerning the $3448.5 \mathrm{meV}$, the evolution of its intensity with temperature follows that of $\mathrm{I}_{2}$, confirming its excitonic nature. Moreover, since the LO-phonon energy in $\mathrm{GaN}$ is $91 \mathrm{meV},{ }^{20}$ this line cannot be assigned to a LO-phonon replica of excitonic lines. Weak $E_{2}$-phonon-assisted replicas are sometimes observed $17.6 \mathrm{meV}$ below the $\mathrm{I}_{2}$ line, ${ }^{20,21}$ which is far from the energy separation of $21.5 \pm 0.3 \mathrm{meV}$ between the $\mathrm{I}_{2}$ and $3448.5 \mathrm{meV}$ lines.

The origin of the $3448.5 \mathrm{meV}$ transition is yet to be ascertained, in spite of several PL studies carried out on similar types of structures. ${ }^{6,22,23}$ The position of this line relative to the $\mathrm{I}_{2}$ line coincides with the so-called two-electron satellites (TES) of the donor-bound exciton line, in agreement with several reports. ${ }^{17,19,21,24,25}$ Contrary to Ref. 23, we discard the possibility that this line corresponds to the so-called $Y_{1}$ transition assigned to inversion domains in $\mathrm{N}$-face $\mathrm{GaN},{ }^{26}$ although it is well known that MBE growth may result in $\mathrm{N}$-face GaN. As a matter of fact, recent studies by convergent beam electron diffraction have established that $\mathrm{GaN}$ $\mathrm{NCs}$, grown by us or other groups by MBE, do grow with Ga polarity. ${ }^{27,28}$ In addition, high-resolution transmission electron microscopy measurements have established that our NCs contain no extended defects (Fig. 3), thus no inversion domains. Finally, the energy position of the line we observe here does not show any energy shift with the excitation density [see Fig. 9b in Ref. 6], unlike what is reported for the $Y_{1}$ line. ${ }^{26}$ Therefore, we assign the $3448.5 \mathrm{meV}$ luminescence to the TES.

As a reminder, the origin of the TES is a kind of Auger

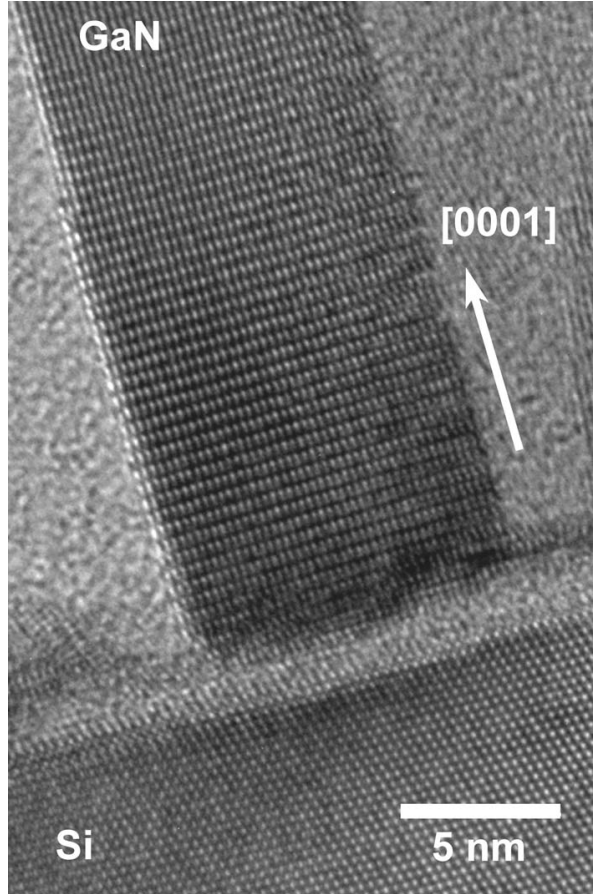

FIG. 3. Cross-sectional TEM image of a GaN NC epitaxially aligned to the $\mathrm{Si}(111)$ substrate

process, internal to the neutral donor-bound exciton. In this process, upon recombination of one electron with the hole, the remaining electron is promoted to some excited state of the neutral donor. In other words, the $\mathrm{I}_{2}$ line results from the following recombination:

$$
\mathrm{D}^{\circ} \mathrm{X}(0) \rightarrow \mathrm{D}_{n=1}^{\circ}+h \nu_{\mathrm{I}_{2}},
$$

where $\mathrm{D}^{\circ} \mathrm{X}(0)$ stands for the ground state of the donor-bound exciton and $\mathrm{D}_{n=1}^{\circ}$ denotes the final state of the transition, which is here the ground state $(1 s)$ of the neutral donor. However, there is some probability that the neutral donor be left in one excited state $(n=2,3$, etc.), giving rise to the TES lines,

$$
\mathrm{D}^{\circ} \mathrm{X}(0, a, b, c \ldots) \rightarrow \mathrm{D}_{(n=2,3, \ldots)}^{\circ}+h \nu_{\mathrm{TES}},
$$

where $0, a, b, c, \ldots$ stands for the ground and so-called rigid rotational excited states ${ }^{21,29-33}$ of the $\mathrm{D}^{\circ} \mathrm{X}$ complex and $n$ $=2,3, \ldots$, represents the different possibilities for the principal quantum number of $\mathrm{D}^{\circ}$ final state. It is important to remark that the relative probabilities of the different recombination channels are very sensitive to the symmetries of both the initial and final states, as recently demonstrated by the detailed spectroscopic study of high-quality $\mathrm{GaN} .{ }^{21}$ In particular, the final state can be a $p$-state of the neutral donor, toward which the recombination is favored if the initial state is the first excited state $\left(a\right.$-state) of the $\mathrm{D}^{\circ} \mathrm{X}$. Such excited states of the $\mathrm{D}^{\circ} \mathrm{X}$ in $\mathrm{GaN}$ have been studied both experimentally ${ }^{29,31,32}$ and theoretically, ${ }^{33}$ being of great importance to explain the temperature dependence of TESrelated PL spectra. ${ }^{21}$ Indeed, when the temperature is raised above typically $5 \mathrm{~K}$, the thermal population of excited states (e.g., the $a$-state) of the $\mathrm{D}^{\circ} \mathrm{X}$ makes the transition to the $2 p$ state of the $\mathrm{D}^{\circ}$ more probable than the transition to the $2 s$ 

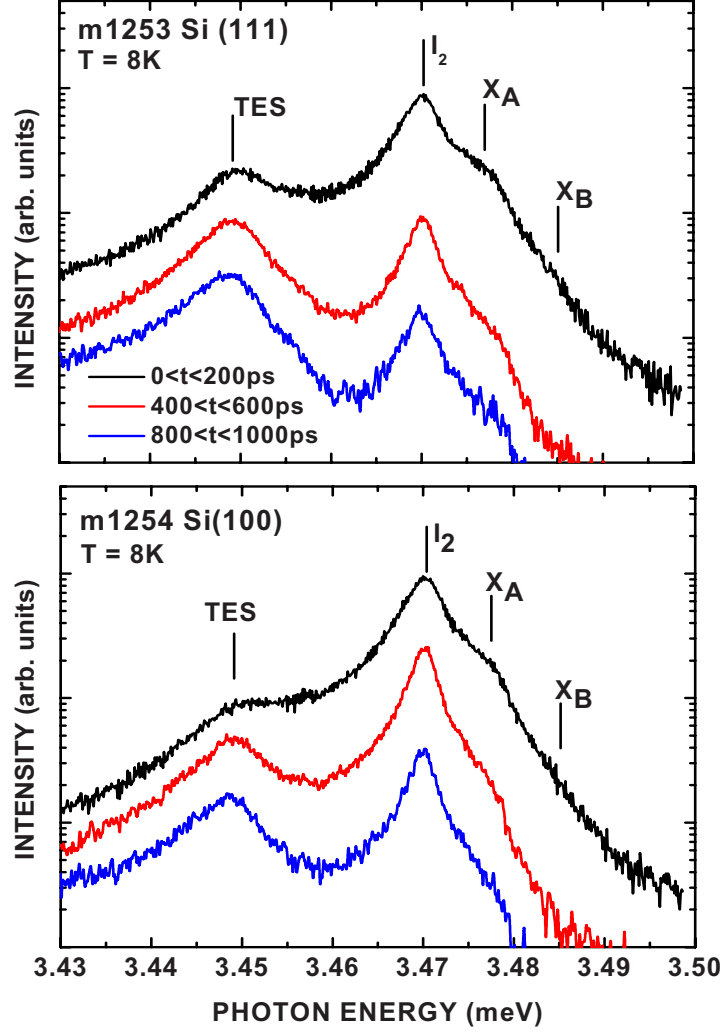

FIG. 4. (Color online) Evolution with time of PL spectra at $T=8 \mathrm{~K}$ of samples $\mathrm{m} 1253$ and $\mathrm{m} 1254$. The domination at short delays of the $\mathrm{I}_{2}$ line is stronger for sample m1254. From the high-energy side of the spectrum, we estimate a carrier temperature of $35 \mathrm{~K}$, at short delays, then slightly decreasing with time.

state. This results in the dominance of the so-called $\mathrm{D}^{\circ} \mathrm{X}$ (a) : $2 p$ transition lying $1.3 \mathrm{meV}$ above the $\mathrm{D}^{\circ} \mathrm{X}(0): 2 s^{21}$

As a rough approximation, the energy difference between the $\mathrm{I}_{2}$ and TES transitions should be close to the energy difference between the first excited $\mathrm{D}_{n=2}^{\circ}$ and ground $\mathrm{D}_{n=1}^{\circ}$ of the neutral donor since the initial states of $\mathrm{D}^{\circ} \mathrm{X}$ are either the same or extremely close in energy. Following the hydrogenic effective-mass approximation, ${ }^{34}$ the TES and $\mathrm{I}_{2}$ lines are therefore separated by $3 / 4$ of the donor binding energy. In our case, the separation of $21.5 \mathrm{meV}$, multiplied by $4 / 3$, yields the effective-mass donor binding energy of $28.7 \mathrm{meV}$, which is very close to the one commonly reported for GaN. ${ }^{17}$ This supports our assignment and is quite consistent with the true binding energies for $\mathrm{Si}$ and $\mathrm{O}$ donors which, accounting for anisotropy of the material, are rather of 30.4 and $33.2 \mathrm{meV}$, respectively. ${ }^{21}$

In the present experiments, the sample temperature was set to $8 \mathrm{~K}$, but the analysis of the high-energy part of the TR spectra (Fig. 4) indicates an electronic temperature of $35 \mathrm{~K}$, in this case. Following the work of Paskov et al., ${ }^{21}$ we should therefore expect the TES to be dominated by a $\mathrm{D}^{\circ} \mathrm{X}(a): 2 p$ (or even higher excited) transition. The measured energy separation of $21.5 \mathrm{meV}$ is then compatible with a $\mathrm{Si}^{\circ} \mathrm{X}_{A}(a): 2 p$ transition and consequently with an $\mathrm{I}_{2}$ line dominated by the $\mathrm{Si}^{\circ} \mathrm{X}_{A}: 1 s$ transition. We then tentatively ascribe the weak line that appears on the low-energy side of the $\mathrm{I}_{2}$ line between 15 and $45 \mathrm{~K}$ to the $\mathrm{O}^{\circ} \mathrm{X}_{A}: 1 s$ transition. $^{21}$

In spite of these assignments, two points remain to be explained: (i) the above-mentioned large linewidths, unusual for such a high-quality unstrained $\mathrm{GaN}$ and (ii) the unusually high relative intensity of the TES line, in comparison to the $I_{2}$. As a matter of fact, the intensity ratio between $I_{2}$ and the TES in GaN compact layers is quite commonly of the order of 100 (Refs. 17 and 29) or 30-50, ${ }^{21}$ depending on the donor involved. In our samples, the TI- (or continuous wave) PL intensities of these lines show, instead, a ratio comprised between 0.7 and 6. From the TR-PL experiments (see Table I) these ratios at $t=0$ range between 1 and 9 , typically. We propose below that these two features are both related to the NC geometry of our structures, through surface-related effects.

First, we observe for both $\mathrm{I}_{2}$ and TES a typical FWHM of $5 \mathrm{meV}$. Yet, the studied samples exhibit a very high crystalline quality and are unstrained. ${ }^{8}$ Since these structures present a large surface-to-volume ratio, we believe that surface effects have to be taken into account. To support this suggestion, we remark that the energy and the wave function of the $n=1$ level of a donor are indeed perturbed if the donor and the surface are separated by less than the donor Bohr radius, $a_{D}(3 \mathrm{~nm}$ for $\mathrm{GaN})$. Ideally, if the donor is placed at the surface of a solid, ${ }^{35}$ the effective Rydberg energy is reduced by a factor of 4 . Then the wave function for the ground state is equivalent to a $2 p$ state with a single probability lobe elongated perpendicular to the surface. Without calculation, we understand that $n=2$ states, having a radial envelope function decreasing like $\exp \left(-r / 2 a_{D}\right)$, will be affected when the nucleus approaches the surface by typically less than $\sim 2 a_{D}$, i.e., $\sim 6 \mathrm{~nm}$ in $\mathrm{GaN}$.

The same reasoning stands for the $\mathrm{D}^{\circ} \mathrm{X}$ complex: the influence of the surface will be significant for a donor-tosurface distance smaller than the typical values for electronnucleus and hole-nucleus distances. Suffczynski and Wolniewicz $^{36}$ calculated these distances in units of $a_{D}$, as a function of the ratio of electron and hole effective masses. For $\mathrm{GaN}$, the hole dispersion relation is anisotropic and $m_{e}^{*} / m_{h}^{*}$ is comprised between 0.2 and $0.4,{ }^{37,38}$ which allows us to estimate, for the $\mathrm{D}^{\circ} \mathrm{X}$ ground state, the average distance of the hole (of any of the two electrons) to the donor nucleus as $\left\langle r_{h}\right\rangle=6.3 \pm 0.7 \mathrm{~nm}\left(\left\langle r_{e}\right\rangle=3.7 \pm 0.1 \mathrm{~nm}\right)$. The interelectron average distance is estimated at $\left\langle r_{12}\right\rangle=6.7 \pm 0.3 \mathrm{~nm}$.

According to these values, when the donor nucleus lies within a surface layer some $\sim 7 \mathrm{~nm}$ thick, the energies of the ground and excited states of the $\mathrm{D}^{\circ} \mathrm{X}$ and of the $\mathrm{D}^{\circ}$ are modified. Therefore, since the donor positions are statistically distributed in this surface layer, the transition energies involving these donors are necessarily distributed too. For NCs with a radius of $15 \mathrm{~nm}(30 \mathrm{~nm})$, the volume of this surface layer constitutes $71 \%(41 \%)$ of the total volume. Thus, the random distribution of donor sites could be an intrinsic origin of the broadening of all PL lines involving donors. Notice that in a typical PL experiment on GaN compact layers, the penetration depth of the excitation laser is about 100-200 nm, thus reducing the surface-layer volume to $3 \%-7 \%$ of the overall probed volume.

Concerning the unusually intense TES lines, we remark that the same surprising result has been reported for $\mathrm{ZnO}$ nanocolums. ${ }^{39}$ This suggests, once more, that the proximity 
of donor nuclei from the surface may deform enough the $\mathrm{D}^{\circ} \mathrm{X}$ and $\mathrm{D}^{\circ}$ envelope functions to perturb profoundly the relative probabilities of the different allowed transitions. In other words, we believe that the favored recombination channels of donor-bound excitons depend on the position of the donors in the columns. A donor situated in the core of the NC is equivalent to the one in regular epilayers, i.e., the $\mathrm{D}^{\circ} \mathrm{X}$ recombines preferentially into the $\mathrm{D}_{n=1}^{\circ}(1 s)$ state and seldom into the $\mathrm{D}_{n=2}^{\circ}$ state. On the other hand, when the donor lies in the surface layer defined above, the necessary deformation of envelope functions of all states for the initial $\mathrm{D}^{\circ} \mathrm{X}$ and for the final $\mathrm{D}^{\circ}$ would increase the relative probability of involving excited states of the donor.

\section{TIME-RESOLVED PHOTOLUMINESCENCE}

TR-PL measurements have been carried out in NCs grown on $\mathrm{Si}(111)$ and $\mathrm{Si}(100)$ in order to get a better understanding of the dynamics of the $\mathrm{I}_{2}$ and the TES. In a simple approach, both TES and $\mathrm{I}_{2}$ are expected to exhibit the same decay rates: ${ }^{40}$ Assuming that the initial states are the same for both transitions, the density of donor-bound excitons should, indeed, follow:

$$
n_{\mathrm{D}^{\circ} \mathrm{X}}(t)=n_{0} \exp \left[-t\left(\tau_{1}+\tau_{2}\right) / \tau_{1} \tau_{2}\right],
$$

where $\tau_{1}=\tau_{n=1}$ and $\tau_{2}=\tau_{n=2}$ are the decay times for the processes involving the $1 s$ and the $2 s / 2 p$ states of the donor, respectively. Higher excited states of the neutral donor should also be considered as possible final states, and Eq. (3) can easily be generalized to that case, without changing the fact that all lines should, in principle, follow the same dynamics. The situation is in fact more complex because the favored initial states (rotator excited states) for the various observed transitions may not be exactly the same, for the $2 s$ and $2 p$ final states. In addition, even when the initial states are the same, Paskov et al. ${ }^{21}$ observed that the TES $\left(\mathrm{D}^{\circ} \mathrm{X}: 2 s\right)$ shows a slower decay than the $\mathrm{I}_{2}\left(\mathrm{D}^{\circ} \mathrm{X}_{A}: 1 s\right)$ line. They attributed this unexpected behavior to the possibility of different sites for the donor nucleus, where the respective dynamics of TES and $\mathrm{I}_{2}$ lines could be different.

In our NC structures we also observe that the apparent decay $\tau_{\mathrm{PL}}$ (TES) of the TES luminescence is always slower than that of $\mathrm{I}_{2}$, which we denote $\tau_{\mathrm{PL}}\left(\mathrm{I}_{2}\right)$ (see Table I, Figs. 4 and 5). This is why the PL signal of the TES dominates the spectrum after a few hundreds of picoseconds, for sample m1253. However, this results not only from a slower decay, but also from the strong relative intensity of the TES line at short delays. The decay dynamics observed here are somewhat faster than those reported, e.g., in Ref. 21. Similar to previous reports for high-quality GaN epilayers, ${ }^{25}$ the free exciton decay is fast and nonexponential in our NCs. There is a clear correlation between its initial decay rate and the rising dynamics of the $\mathrm{I}_{2}$ and TES lines. The slower the free exciton dynamics (e.g., sample m1254) the longer the rise time of the two donor-related excitonic recombinations. Moreover, after some transient phase, the $\mathrm{I}_{2}$ and $\mathrm{X}_{A}$ line adopt the same recombination dynamics.

The condition for the $\mathrm{D}^{\circ} \mathrm{X}$ to show such rise time and delayed maximum compared to $\mathrm{X}_{A}$ is that the capture rate of

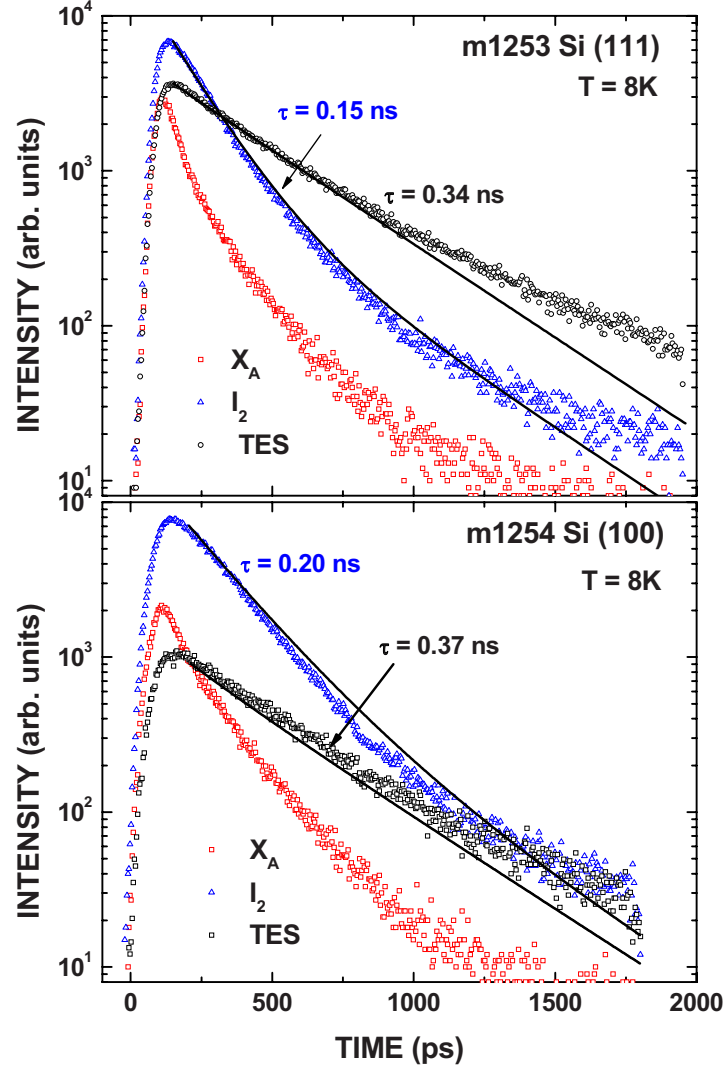

FIG. 5. (Color online) Time dependence of PL intensities of the $\mathrm{X}_{A}, \mathrm{I}_{2}$, and TES transitions at $T=8 \mathrm{~K}$ for samples $\mathrm{m} 1253$ and $\mathrm{m} 1254$. Decay time constants have been obtained by single-exponential fitting of the appropriate zones. Solid curves are the results of the two-zone (core-shell) model.

excitons by donors is faster than the $\mathrm{D}^{\circ} \mathrm{X}$ decay. Then, the fact that the two decays $\left(\mathrm{X}_{A}\right.$ and $\left.\mathrm{D}^{\circ} \mathrm{X}\right)$ are perfectly parallel suggests that bound and free exciton states are thermalized. This means that thermal energy is sufficient to permit an instantaneous exchange of carriers between the two states. The actual temperature of $35 \mathrm{~K}$ is compatible with this hypothesis, yielding a thermal free-to-bound exciton population ratio of $10 \%$.

Concerning the main topic of this paper, i.e., the related dynamics of the $I_{2}$ and TES lines, it is clear (Fig. 6) that their initial states, when not identical, are thermalized, for $T$ $=35 \mathrm{~K}$, being separated by $\sim 1 \mathrm{meV}^{30-32}$ Therefore, their respective radiative lifetimes should control their instanta-

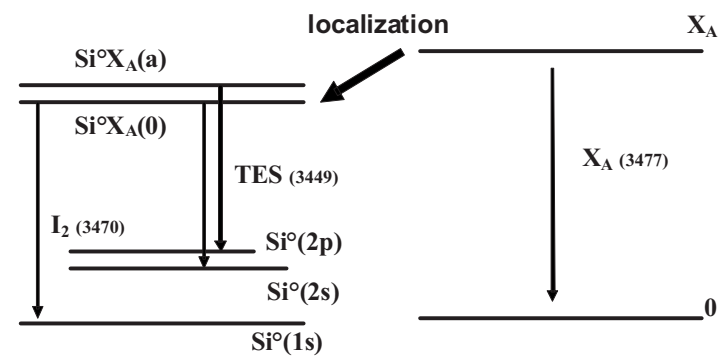

Neutral donor bound exciton

Free exciton

FIG. 6. Schematic view of the different excitonic transitions involved in our study. Energies are expressed in $\mathrm{meV}$. The energy difference between $\mathrm{Si}^{\circ} \mathrm{X}_{A}(a)$ and $\mathrm{Si}^{\circ} \mathrm{X}_{A}(0)$ states is of the order of $1 \mathrm{meV}$ (Refs. 25-27). 
neous intensity ratio at $t=0$, but their time decays should in principle be parallel. Since the latter is not observed, we propose a model involving two regions of the NCs having quite different intrinsic characteristics.

\section{MODELING AND DISCUSSION}

This approach is inspired by the suggestion of Paskov et $a l^{21}$ that the intrinsic characteristics of $\mathrm{D}^{\circ} \mathrm{X} \rightarrow \mathrm{D}^{\circ}$ transitions may depend on the donor position. This is here justified by the fact that we know that the wave functions of $\mathrm{D}^{\circ} \mathrm{X}$ and $\mathrm{D}^{\circ}$ states will strongly depend on the position of the donor nucleus along the radius of the NC. Therefore we choose to define two regions: a core $(c)$ and a shell $(s)$, where the relative probabilities of the $\mathrm{I}_{2}$ and TES transitions differ substantially. Actually the situation is certainly more complex, with some progressive variation of properties from the core toward the surface of the NC, and thus we should consider the following model as qualitative.

We write separately the rate equations for the populations of $\mathrm{D}^{\circ} \mathrm{X}$ located in the core and in the shell of the NC. We call $\alpha$ the volume fraction occupied by the bulklike core (c) of the $\mathrm{NC}$, where the $\mathrm{D}^{\circ} \mathrm{X} \rightarrow \mathrm{D}^{\circ}$ recombination is the same as in good quality bulk GaN. On the other hand, the $\mathrm{D}^{\circ} \mathrm{X}$ and $\mathrm{D}^{\circ}$ states are affected by the surface in the shell $(s)$ region defined by the volume fraction $(1-\alpha)$. For example, for a $\mathrm{NC}$ with radius of $30 \mathrm{~nm}$ and assuming a shell layer thickness of $7 \mathrm{~nm}$, we find $\alpha=0.59$. In both regions, the time decay of $\mathrm{D}^{\circ} \mathrm{X}$ density follows Eq. (3) but with different values of $\tau_{1}=\tau_{1 s}$ and $\tau_{2}=\tau_{n=2}$, for the $(c)$ and $(s)$ regions. Again, we have restricted the model to $n=2$ states, for simplification.

In our low-temperature experiments, increasing the surface-to-volume ratio of NCs does not induce any decrease in the emission intensity. We therefore conclude that, if enhanced surface-related nonradiative recombination would take place, it would equally affect the dynamics and intensities of both core and surface regions. For the present donorbound excitons (essentially localized) and given the low temperature, we therefore neglect nonradiative recombination in our model.

The intensities of $\mathrm{I}_{2}$ and TES for the whole $\mathrm{NC}$ are given by the weighted sums of contributions from the core, with weight $\alpha$, and from the shell, with weight $(1-\alpha)$. These contributions for each region are given by

$$
\begin{aligned}
& I_{\mathrm{I}_{2}(c, s)}(t) \propto\left[\tau_{1(c, s)}\right]^{-1} \exp \left[-t / \tau_{\mathrm{eff}}(c, s)\right], \\
& I_{\mathrm{TES}(c, s)}(t) \propto\left[\tau_{2(c, s)}\right]^{-1} \exp \left[-t / \tau_{\mathrm{eff}}(c, s)\right] .
\end{aligned}
$$

In Eq. (4), the same decay times $\tau_{\operatorname{eff}(c, s)}$ are taken for the TES and $I_{2}$ transitions, according to the above remark that their initial states are thermally indistinguishable, if not identical. Nevertheless, the instantaneous intensities of these transitions are related to two different radiative decay times. For bulk GaN, the TES is about 50 times less intense $\mathrm{e}^{21}$ than the $\mathrm{I}_{2}$. We thus can limit the number of adjustable parameters by setting the ratio of radiative lifetimes, for the core region to $\eta=\tau_{2(c)} / \tau_{1(c)}=50$. However, this value corresponds to the ratio measured at $T=2 \mathrm{~K}$ in Ref. 21 for Si donors in a high- quality GaN epilayer. For the present $T=35 \mathrm{~K}$, in the presence of both $\mathrm{Si}$ and $\mathrm{O}$ atoms, a larger variety of initial and final states are involved in the observed TES recombinations and we know ${ }^{21}$ that the relative probabilities of the different TES contributions are therefore drastically affected. Consequently, $\eta$ should in principle be affected, too, but here it was kept constant, for simplification.

In the NCs, the intensity ratio between $\mathrm{I}_{2}$ and TES is much smaller than in bulk GaN, which suggests that the TES PL contribution mainly arises from the shell region. Then, the apparent decay rate of the TES line can be approximated by

$$
\tau_{\mathrm{PL}}(\mathrm{TES}) \approx \tau_{\mathrm{eff}(s)}=\tau_{1(s)} \tau_{2(s)} /\left(\tau_{1(s)}+\tau_{2(s)}\right),
$$

and we can simplify the intensity ratio, at $t=0$, between the $\mathrm{I}_{2}$ and TES lines,

$$
\rho=\frac{I_{\mathrm{I}_{2}}(0)}{I_{\mathrm{TES}}(0)} \approx \frac{\tau_{2(s)}}{1-\alpha}\left(\frac{\alpha}{\tau_{1(c)}}+\frac{1-\alpha}{\tau_{1(s)}}\right) .
$$

As shown in Figs. 4 and 5 and in Table I, we have experimental values for this ratio, as well as for the effective PL decay times for the TES. The time decay of the $\mathrm{I}_{2}$ line is slightly nonexponential, but we can measure a time constant at short delay, also reported in Table I for all samples. Starting from these experimental data and using the approximations in Eqs. (5) and (6), we calculate the time evolution of the overall $\mathrm{D}^{\circ} \mathrm{X}$ population and of the intensity of $\mathrm{I}_{2}$ and TES lines. For this simplified model, the solution is analytical and both decays are biexponential,

$$
I_{\mathrm{I}_{2}}(t)=\alpha\left(\tau_{1(c)}\right)^{-1} \exp \left(-t / \tau_{A}\right)+(1-\alpha)\left(\tau_{2(c)}\right)^{-1} \exp \left(-t / \tau_{B}\right),
$$

$I_{\mathrm{TES}}(t)=\alpha\left(\tau_{1(s)}\right)^{-1} \exp \left(-t / \tau_{A}\right)+(1-\alpha)\left(\tau_{2(s)}\right)^{-1} \exp \left(-t / \tau_{B}\right)$.

with decay times $\tau_{A}$ and $\tau_{B}$ given by

$$
\begin{aligned}
& \tau_{A}=\left[\frac{\alpha}{1-\alpha}\left(\frac{1}{\tau_{1(c)}}-\frac{1}{\tau_{2(c)}}\right)+\frac{1}{\tau_{2(s)}}+\frac{1}{\tau_{1(s)}}\right]^{-1}, \\
& \tau_{B}=\tau_{\mathrm{eff},(s)}=\tau_{1(s)} \tau_{2(s)} /\left(\tau_{1(s)}+\tau_{2(s)}\right) .
\end{aligned}
$$

By using Eqs. (7) and (8), we can fit the experimental decays and therefore extract relevant values for parameters of interest, namely, $\tau_{1(c)}, \tau_{2(c)}, \tau_{1(s)}, \tau_{2(s)}$, and $\alpha$. In Fig. 5, solid lines show the best fitting calculated curves for samples $\mathrm{m} 1253$ and $\mathrm{m} 1254$. The values of the fitting parameters are gathered in Table I, for all samples.

The quality of the fitting is satisfactory, given the simplicity of the model. This confirms that we can simultaneously describe the different decay dynamics, as well as the intensity ratios of the TES and $\mathrm{I}_{2}$ emission lines, by accounting for the existence of core and shell regions. The different decay times resulting from the fitting do not present, however, the same degree of uncertainty: "dominant" times, such as $\tau_{1(c)}$ and $\tau_{2(s)}$ are necessarily very close to the experimental decay times $\tau_{\mathrm{PL}}(\mathrm{TES})$ and $\tau_{P L}\left(\mathrm{I}_{2}\right)$, respectively, and they are therefore estimated rather accurately. On the other hand, $\tau_{2(c)}$ and $\tau_{1(s)}$ present a much larger uncertainty, being "non- 
dominant" and because of the strong hypothesis of the fixed ratio $\tau_{1(c)} / \tau_{2(c)}$. For this reason $\tau_{1(s)} / \tau_{2(s)}$ is determined with poor accuracy. Therefore it is difficult to compare it with its counterpart in the core region and to discuss its apparent variation with, e.g., the $\mathrm{NC}$ diameter, especially considering the simplicity of the two-region model and approximations such as Eq. (6).

It seems interesting to compare our fitting decay times in the core region (see Table I) with those measured, e.g., by Paskov et $a .^{21}$ for a high-quality GaN epilayer. The present $\tau_{1(c)}$ and $\tau_{2(c)}$ are clearly shorter, which can be related to the temperature of $35 \mathrm{~K}$ that we estimate. Indeed, as shown by Eq. (3), including more allowed recombination channels (by thermal excitation, here) necessarily shortens the effective decay time of $\mathrm{D}^{\circ} \mathrm{X}$ population. By the way, it is difficult to know if we should compare our $\tau_{1(c)}$ (typically $0.2 \mathrm{~ns}$ ) to the value of $\sim 0.5 \mathrm{~ns}$ measured for $\mathrm{I}_{2}$ in Ref. 21 , or if it is our $\tau_{2(c)}$ (typically 1 or a few nanoseconds, with large uncertainty) that should be compared to the 1.1 and $1.8 \mathrm{~ns}$, measured, ${ }^{21}$ respectively, for $\mathrm{SiX}_{A}: 2 s$ and $\mathrm{OX}_{A}: 2 s$ transitions since, as mentioned in Ref. 21, these different dynamics precisely correspond to different donor sites.

In summary, we prefer to consider that the nondominant fitting characteristic times do not vary substantially nor monotonously among the four samples. Rather than being free parameters, they are related to dominant times by strong constraints imposed to the model: $\tau_{1(c)}$ has to be close to the overall decay time measured for $\mathrm{I}_{\mathrm{I} 2}$, and $\tau_{1(s)}$ and $\tau_{2(s)}$ are related to the experimental decay time of the TES by Eqs. (5) and (8). Nevertheless, the core and shell time constants are not independent because of the constraint on the intensity ratio given by Eq. (6).

This ratio is measured with high precision in our experiments and, in Eq. (6), it mainly involves the dominant times $\tau_{1(c)}$ and $\tau_{2(s)}$ together with the volume-fraction parameter $\alpha$. This is why, instead of imposing a value for $\alpha$, we used it as a fitting parameter, necessarily estimated with a good accuracy. We found that $\alpha$ generally increases with the average $\mathrm{NC}$ radius. Despite the very large size dispersion of the NCs and the crudeness of the model, this trend is quite clear. Given the measured NC radii, the obtained values for $\alpha$ are compatible with a shell layer thickness in the range of 8-9 $\mathrm{nm}$, which compares quite well with the $7 \mathrm{~nm}$ estimated above, considering typical $\mathrm{D}^{\circ} \mathrm{X}$ or $\mathrm{D}^{\circ}$ state radii.

The consistency of these results supports our interpretation based on the sensitivity of $\mathrm{D}^{\circ} \mathrm{X}$ internal properties to the vicinity of the $\mathrm{NC}$ surface. According to our results, the symmetry breaking induced by this vicinity favors the so-called two-electron transitions to the detriment of to the usually dominant $\mathrm{I}_{2}$ line. Far beyond the scope of the present experimental study, a donor-site dependent model of energies and wave-functions of $\mathrm{D}^{\circ} \mathrm{X}$ and $\mathrm{D}^{\circ}$ states in realistic NCs should be developed to ascertain completely this interpretation.

\section{CONCLUSION}

In summary, we have studied in detail the excitonic PL of GaN NCs grown on silicon substrates. Substrate orientation did not show any significant effect on the optical prop- erties of the NCs, which exhibit free and donor-bound exciton lines. The latter is accompanied by TESs that are unusually intense. We have shown that this intensity increases when the NC average radius decreases. Moreover, the TES recombination dynamics is unexpectedly much slower than the dominant donor-bound exciton recombination. To explain this result, we have developed a core-shell model that relies on donor position dependent dynamics of the two principal donor-related excitonic transitions. The good agreement obtained between the model and experiments confirms that the smaller the $\mathrm{NC}$ radius, the larger the average influence of the surface on excitonic transitions. We believe that this influence manifests itself through the alteration of the wave functions of the $\mathrm{D}^{\circ} \mathrm{X}$ and $\mathrm{D}^{\circ}$ states involved. This simultaneously explains slight fluctuations in energies - thus large linewidths - and modified relative probabilities for the $\mathrm{I}_{2}$ and TES recombinations. Thorough calculations of all quantum states of $\mathrm{D}^{\circ} \mathrm{X}$ and $\mathrm{D}^{\circ}$ as a function of the distance to the NC surface are needed to quantitatively support our conclusions.

\section{ACKNOWLEDGMENTS}

The authors wish to thank M. Leroux, T. Guillet, and T. Taliercio for enlightening comments and R. Rochat for the technical support. IPEQ-EPFL group acknowledges support from the Swiss National Science Foundation and from the Swiss NCCR research program Quantum Photonics. GES group was supported by the Nanosciences French National Program through the "BUGATI" project. E.C. would also like to acknowledge financial support from the Spanish Ministry of Education (Grant No. NAN04/09109/C04/2, Consolider-CSD 2006-19) and the Community of Madrid (Grant Nos. GR/MAT/0042/2004 and S-0505/ESP-0200).

${ }^{1}$ S. Nakamura, T. Mukai, and M. Senoh, Appl. Phys. Lett. 64, 1687 (1994). ${ }^{2}$ S. D. Lester, F. A. Ponce, M. G. Craford, and D. A. Steigerwald, Appl. Phys. Lett. 66, 1249 (1995).

${ }^{3}$ J. C. Johnson, H.-J. Choi, K. P. Knutsen, R. D. Schaller, P. Yang, and R.J. Saykally, Nature Mater. 1, 106 (2002).

${ }^{4}$ Z. Zhong, F. Qian, D. Wang, and C. M. Lieber, Nano Lett. 3, 343 (2003). ${ }^{5}$ M. C. McAlpine, R. S. Friedman, S. Jin, K. Lin, W. U. Wang, and C. M. Lieber, Nano Lett. 3, 1531 (2003).

${ }^{6}$ E. Calleja, M. A. Sánchez-Garciá, F. J. Sánchez, F. Calle, F. B. Naranjo, E. Muñoz, U. Jahn, and K. Ploog, Phys. Rev. B 62, 16826 (2000).

${ }^{7}$ J. E. Van Nostrand, K. L. Averett, R. Cortez, J. Boeckl, C. E. Stutz, N. A. Sanford, A. V. Davydov, and J. D. Albrecht, J. Cryst. Growth 287, 500 (2006).

${ }^{8}$ J. Sánchez-Páramo, J. M. Calleja, M. A. Sánchez-García, E. Calleja, and U. Jahn, Physica E (Amsterdam) 13, 1070 (2002).

${ }^{9}$ H.-M. Kim, D. S. Kim, D. Y. Kim, T. W. Kang, Y.-H. Cho, and K. S. Chung, Appl. Phys. Lett. 81, 2193 (2002).

${ }^{10}$ M. He and S. Noor Mohammad, J. Vac. Sci. Technol. B 25, 1909 (2007).

${ }^{11}$ J. Ristić, C. Rivera, E. Calleja, S. Fernández-Garrido, M. Povoloskyi, and A. Di Carlo, Phys. Rev. B 72, 085330 (2005).

${ }^{12}$ J. Ristić, E. Calleja, A. Trampert, S. Fernández-Garrido, C. Rivera, U. Jahn, and K. H. Ploog, Phys. Rev. Lett. 94, 146102 (2005).

${ }^{13}$ L. Cerutti, J. Ristić, S. Fernández-Garrido, E. Calleja, A. Trampert, S. Lazic, and J. M. Calleja, Appl. Phys. Lett. 88, 213114 (2006).

${ }^{14}$ J. Ristić, M. A. Sánchez-García, E. Calleja, A. Pérez-Rodriguez, C. Serre, A. Romano-Rodríguez, J. R. Morante, V. R. Koegler, and W. Skorupa, Mater. Sci. Eng., B 93, 172 (2002).

${ }^{15}$ R. Meijers, T. Richter, R. Calarco, T. Stoica, H.-P. Bochem, M. Marso, and H. Lüth, J. Cryst. Growth 289, 381 (2006).

${ }^{16}$ A. Trampert, J. Ristić, U. Jahn, E. Calleja, and K. H. Ploog, in Proceedings of the 13th International Conference on Microscopy of Semiconduct- 
ing Materials, edited by A. G. Cullis and P. A. Midgley, IOP Conference Series (IOP Publishing Ltd., Dirac House, Temple Back, Bristol, England, 2003), Vol. 180, p. 167.

${ }^{17}$ K. Kornitzer, T. Ebner, K. Thonke, R. Sauer, C. Kirchner, V. Schwegler, M. Kamp, M. Leszczynski, I. Grzegory, and S. Porowski, Phys. Rev. B 60, 1471 (1999).

${ }^{18}$ B. Monemar, J. Mater. Sci.: Mater. Electron. 10, 227 (1999).

${ }^{19}$ J. A. Freitas, W. J. Moore, B. V. Shanabrook, G. C. B. Braga, D. D Koleske, S. K. Lee, S. S. Park, and J. Y. Han, Phys. Status Solidi B 240, 330 (2003)

${ }^{20}$ T. Azuhata, T. Matsunaga, K. Shimada, K. Yoshida, T. Sota, and S. Nakamura, Physica B 219-220, 493 (1996).

${ }^{21}$ P. P. Paskov, B. Monemar, A. Toropov, J. P. Bergman, and A. Usui, Phys. Status Solidi C 4, 2601 (2007).

${ }^{22}$ F. Calle, F. J. Sánchez, J. M. G. Tijero, M. A. Sánchez-García, E. Calleja, and R. Beresford, Semicond. Sci. Technol. 12, 1396 (1997).

${ }^{23}$ L. H. Robins, K. A. Bertness, J. M. Barker, N. A. Sanford, and J. B. Schlager, J. Appl. Phys. 101, 113506 (2007).

${ }^{24}$ M. A. Reshchikov and H. Morkoç, J. Appl. Phys. 97, 061301 (2005)

${ }^{25}$ E. Frayssinet, B. Beaumont, J. P. Faurie, P. Gibart, Zs. Makkai, B. Pécz, P. Lefebvre, and P. Valvin, MRS Internet J. Nitride Semicond. Res. 7, 8 (2002).

${ }^{26}$ M. A. Reshchikov, D. Huang, F. Yun, P. Visconti, L. He, H. Morkoç, J. Jasinski, Z. Liliental-Weber, and R. J. Molnar, J. Appl. Phys. 94, 5623 (2003).
${ }^{27}$ D. Cherns, L. Meshi, I. Griffiths, S. Khongphetsak, S. V. Novikov, N. Farley, R. P. Campion, and C. T. Foxon, Appl. Phys. Lett. 92, 121902 (2008).

${ }^{28}$ A. Trampert, personal communication (May 25, 2005).

${ }^{29}$ B. J. Skromme, J. Jayapalan, R. P. Vaudo, and V. M. Phanse, Appl. Phys. Lett. 74, 2358 (1999).

${ }^{30}$ W. Rühle and W. Klingenstein, Phys. Rev. B 18, 7011 (1978).

${ }^{31}$ G. Neu, M. Teisseire, P. Lemasson, H. Lahreche, N. Grandjean, F. Semond, B. Beaumont, I. Grzegory, S. Porowski, and R. Triboulet, Physica B 302-303, 39 (2001).

${ }^{32}$ G. Neu, M. Teisseire, B. Beaumont, H. Lahreche, and P. Gibart, Phys. Status Solidi B 216, 79 (1999).

${ }^{33}$ B. Gil, P. Bigenwald, M. Leroux, P. P. Paskov, and B. Monemar, Phys. Rev. B 75, 085204 (2007).

${ }^{34}$ T. Skettrup, M. Suffczynski, and W. Gorzkowski, Phys. Rev. B 4, 512 (1971).

${ }^{35}$ J. A. Levine, Phys. Rev. 140, A586 (1965).

${ }^{36}$ M. Suffczynski and L. Wolniewicz, Phys. Rev. B 40, 6250 (1989).

${ }^{37}$ M. Suzuki, T. Uenoyama, and A. Yanase, Phys. Rev. B 52, 8132 (1995).

${ }^{38}$ I. Vurgaftman and J. R. Meyer, J. Appl. Phys. 94, 3675 (2003).

${ }^{39}$ A. Mézy, S. Anceau, T. Bretagnon, P. Lefebvre, T. Taliercio, G.-C. Yi, and J. Yoo, Superlattices Microstruct. 39, 358 (2006).

${ }^{40}$ A. Wysmolek, K. P. Korona, R. Stepniewski, J. M. Baranowski, J. Bloniarz, M. Potemski, R. L. Jones, D. C. Look, J. Kuhl, S. S. Park, and S. K. Lee, Phys. Rev. B 66, 245317 (2002). 